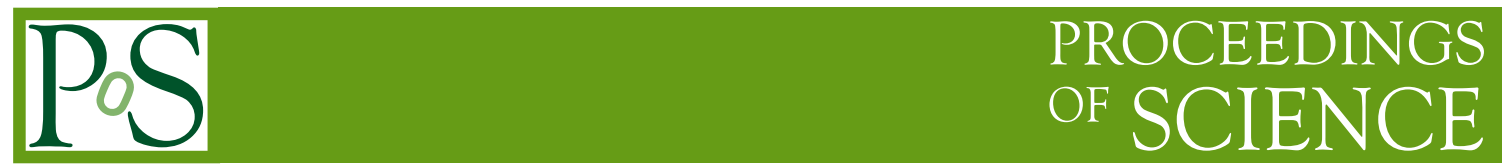

\title{
Telescope Array Lightning Location System
}

\section{Takeshi Okuda*}

Ritsumeikan University

E-mail: okuda@icrr.u-tokyo.ac.jp

\section{for the Telescope Array Collaboration}

The Telescope Array (TA) experiment detects air-showers induced by ultra high energy cosmic rays. The TA ground Surface particle Detector array (TASD) observed some short-duration bursts of air-shower like events. As reported at ICRC2015, these events are evidently correlated with lightning. Therefore, we have deployed detectors for lightning discharge in the vicinity of the TASD. We report the status and study of the Telescope Array Lightning Location System (TALLS). 


\section{Introduction}

Some high energy radiation bursts were observed by the Telescope Array Surface Detector(TASD), and correlated with lightning discharge detected by National Lightning Detection Network [1][2]. In order to investigate these relation between high energy radiation and identified lightning discharge with higher statistics, I constructed lightning location system for TASD.

Telescope Array is located in midwest in Utah, USA (Lat 39.3, Lon -112.9, Alt 1400m). It mainly consists of 3 atmospheric fluorescence telescope stations called FD and 507 ground surface particle detectors called SD, see Figure 1(Left). TASD is dual layer plastic scintillation detector, whose detection area is $3 \mathrm{~m}^{2}$ for each, see Figure 1(Right). The shower trigger judgement of TASD is 3 adjacent detectors which was hit by 3 or more particles in $8 \mu \mathrm{s}$. Once triggered, all non-zero particle waveforms in $32 \pm \mu$ s from triggered time are collected from all detectors [3].
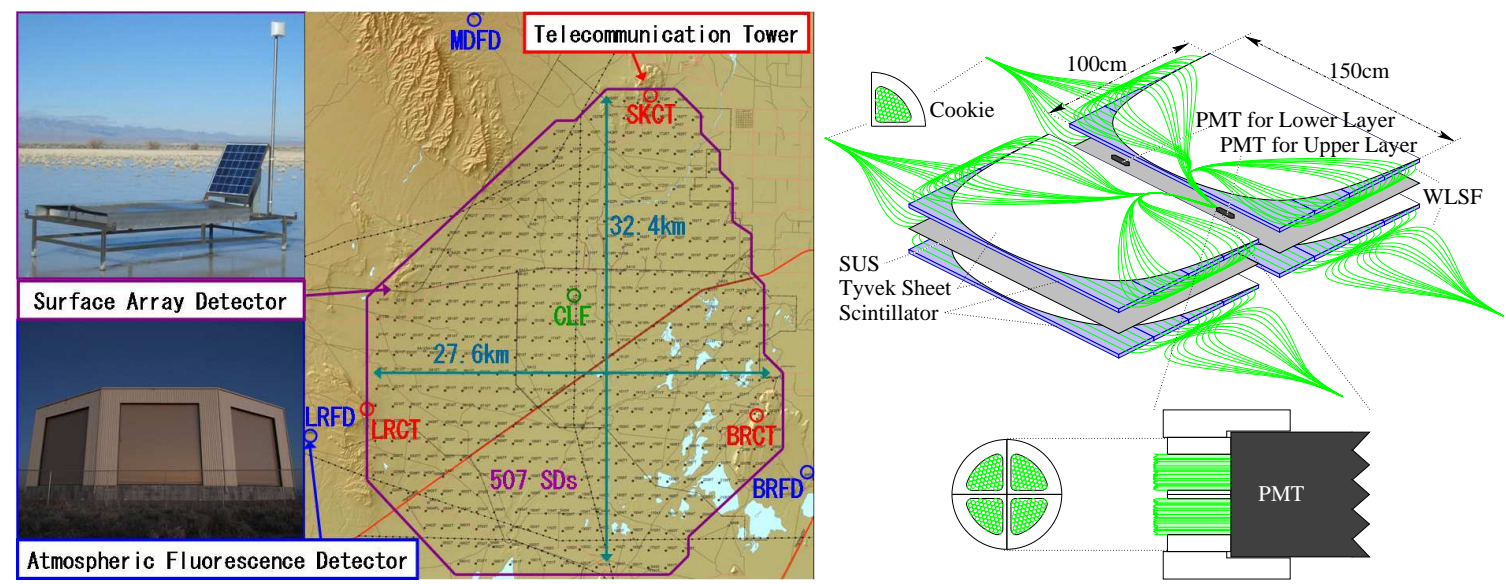

Figure 1: Telescope Array Experiment. Left: 507 ground surface particle detectors (tiny dots above) are surrounded by 3 atmospheric fluorescence telescope stations. Right: Particle detecting part of TASD consists of 2 layers of plastic scintillators. Each scintillation layer has $1.2 \mathrm{~cm}$ thickness and provides information respectively via wavelength-shifting fibers.

There are some precedent observations for the relation between high energy radiation and identified lightning discharge. And the coverage area of precedent observation is several $\mathrm{km}^{2}$ at most, whereas TASD is deployed $680 \mathrm{~km}^{2}$. The radiation detector of precedent observations is mainly NaI. It can measure the radiation energy but the detection area is small and the response is slow. TASD is plastic scintillation detector, It cannot measure the radiation energy but the detection area is more than 100 times larger and the response is 10 times faster. The precedent observations measure the energy of radiation and TASD measures spatial distribution of radiation. Therefore, TASD is unique and complementary to observe high energy radiation from lightning. TASD is running now and located where is stable lightning discharge in summer. In addition, TASD is the largest radiation detector with high sensitivity for electromagnetic component in the northern hemisphere. Near future, there is no promising plan to deploy radiation detectors in such a large area for radiation from lightning. Therefore, I tried to detect lightning discharge at Telescope Array site as many as possible. 


\section{Lightning Location System}

Telescope Array Lightning Location System(TALLS) consists of 4 or 5 lightning detectors (Figure 2). In order to cover TASD, lightning detectors were deployed surrounding TASD. The first detector(LDA) was deployed in September 2014 at Cosmic Ray Center which is workshop for TA, in Delta City where the TA operators usually stay. The second and third detectors(LDB, LDC) were deployed in September 2015 at southern FD stations with solar battery system. The fourth detector(LDD) was deployed in March 2016 at northern FD station with solar battery system. The central data server was placed in Cosmic Ray Center and stored all data from local DAQ-PC beside lightning detector. The local DAQ-PC is controlled via this central data server remotely.
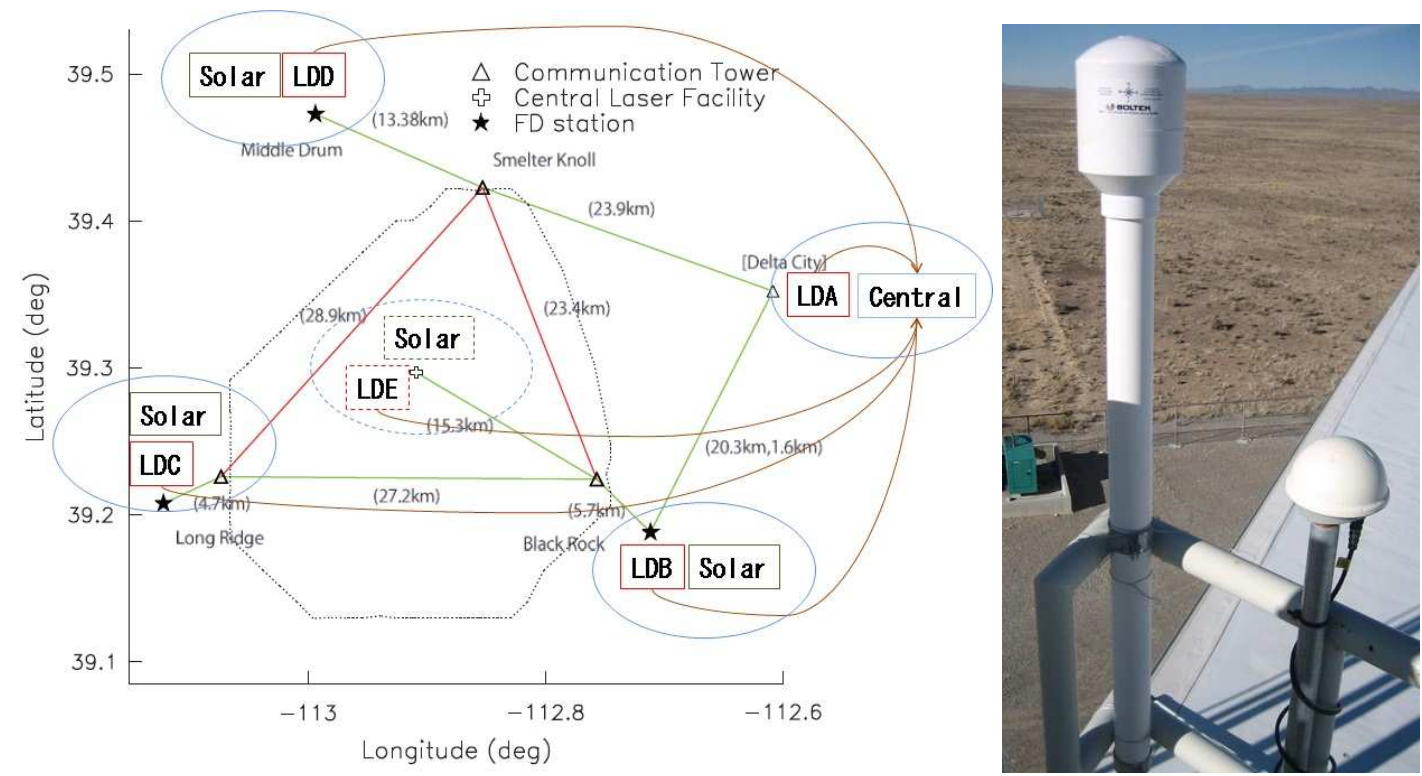

Figure 2: Lightning Location System Left: 4 lightning detectors are located surrounding TASD and running now. LD? shows detector name. Right: Antennas for lightning detector and its GPS on FD station.

The antenna for lightning detector consists of omnidirectional electric field antenna and orthogonal magnetic field loop antennas. Therefore, it outputs 3 waveforms at once. Each waveform has 512 datapoints for $51.2 \mu \mathrm{s}$. Two magnetic field loop antennas are orthogonal, therefore the ratio of the magnetic waveform amplitude indicates the direction of the lightning discharge from the detector. This method is called Magnetic Direction Finder(MDF). In principle, the lightning location by MDF can be determined by 2 detectors at minimum. However, this estimated direction has somewhat large error by the local environment around detector. Therefore, the accuracy of the estimated lightning location is not good. The other method uses the signal arrival timing for each detector. It is called Time Of Arrival(TOA). The time difference of a pair of detectors describe a hyperboloid as candidates of the lightning location. In principle, the lightning location by TOA can be determined by 4 detectors at minimum. This method requires the accurate timing of signal arrival for enough accuracy of the estimated lightning location. Therefore, GPS timing module is used to record the waveform timing. 
As written above, TOA needs 4 detectors to determine the lightning location uniquely at all times. However, TOA with 3 detectors can also determine the lightning location, but sometimes as double root. Even in this case, MDF can be used to choose the proper location from two candidates. Although MDF does not have good accuracy, it is enough to choose one of two. Therefore, TALLS use both method to determine the location for 3-fold coincidence event. Especially, in even case of the malfunction of a detector, TALLS can keep the minimum locating ability.

\section{Observation and Location Determination}

Observation status after fourth detector's deployment is shown in Figure 3. Because the trigger threshold of the detector is set at low level, the trigger rate is more than the data output rate and then the number of alive detector decrease under the huge thunderstorm. These thresholds are adjusted gradually by monitoring the detector status. Basically, these detectors run stably and the installed solar battery systems provide enough power even in winter.

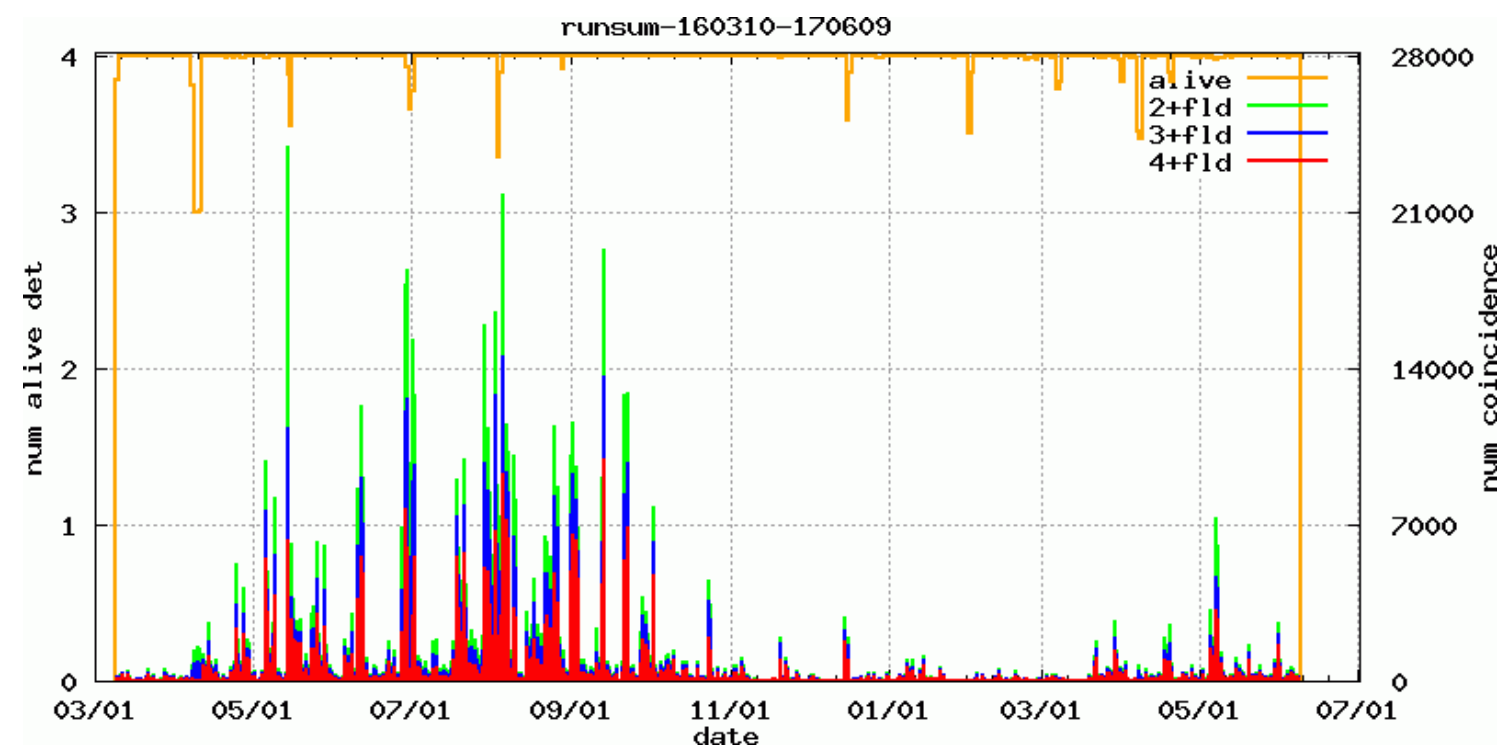

Figure 3: Running Status and the number of daily triggers for 15 months after fourth detector was activated. Orange line shows the number of alive detector calculated in 1 minute resolution. Bars show the number of daily ?-fold triggers.

As an example, I show a 4-fold coincidence event taken on the afternoon of March 22 in 2016, 12 days after the deployment of the fourth detector. The electric field waveforms are almost same shape for 4 detectors whereas the magnetic field waveforms have various amplitude of east-west and north-south components for 4 detectors (Figure 4). The estimated directions by MDF and the hyperbolae by TOA of triggered time are shown in Figure 5. The rough accuracy of MDF can be read in this figure.

\section{Plan and Summary}

The fifth detector(LDE) with solar battery system is ready to deploy to the central laser facility 


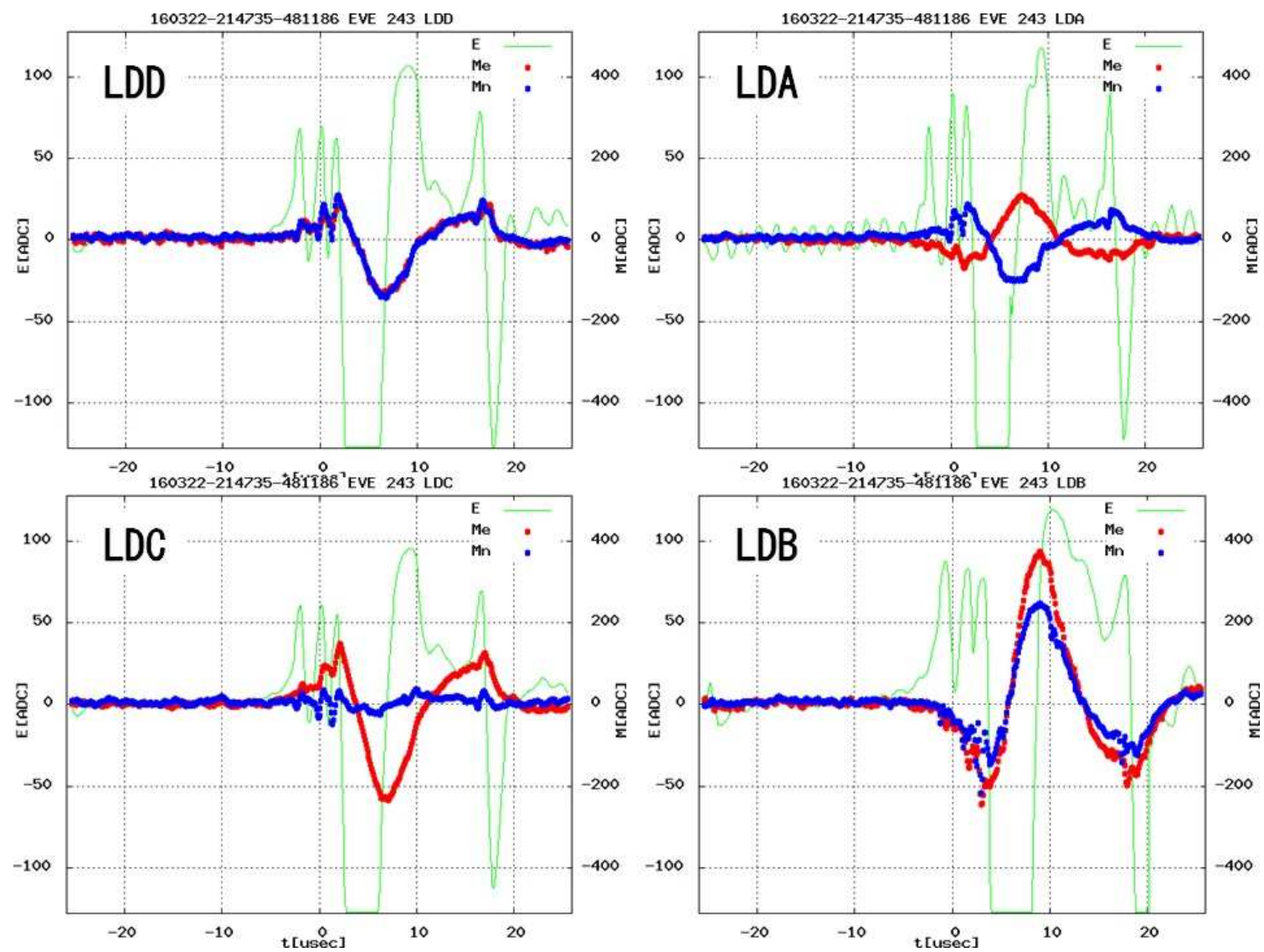

Figure 4: Waveforms for a 4-fold coincidence trigger. Green shows electric field. Red shows east-west magnetic field. Blue shows north-south magnetic field.

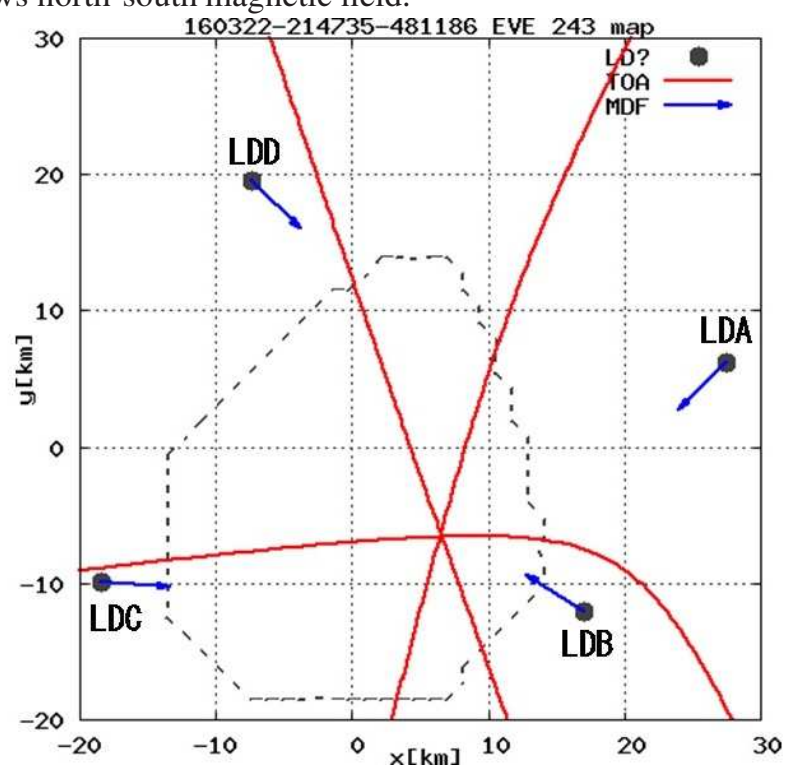

Figure 5: Lightning Location Determination for a 4-fold coincidence trigger. Black dots show the locations of lightning detector. Red lines show hyperbolae by TOA on the basis of LDA. Blue arrows show the directions to the lightning discharge from detector by MDF. 
(Figure 2). However, the construction of expansion of TASD called TAx4 is going on now. There is possibility to deploy the fifth detector for the expanded area, so the location will be decided by near future condition.

MDF can be used to choose proper location in double root but has somewhat large error as written above. The unique location by 4-detectors TOA can be used to evaluate and calibrate MDF. Therefore, many lightning locations by TOA for a long time will be used for this purpose. And I will try to evaluate the peak current of lightning discharge by using amplitudes of the magnetic field waveform and the distances from the detector to the lightning location. Then, of course, I will check the correlation of lightning with TASD data.

Telescope Array Lightning Location System is running from September 2015 stably. The analysis for TALLS is going on.

\section{Acknowledgments}

TALLS is supported by the Mitsubishi Foundation and the joint research program of the Institute for Cosmic Ray Research, University of Tokyo.

\section{References}

[1] T.Okuda, et al., PoS (2015), ICRC2015, 298

[2] R.U.Abbasi, et al., Phys. Lett. A (2017), 381, p2565-2572

[3] T.AbuZayyad, et al., NIM Phys. Res. A (2012), 689, p87-97 